\title{
Improvement of Injection Accuracy in the Media Changing Process for Cell Processing
}

Ryosuke Nonoyama ( $\nabla$ nonoyama@kokushikan.ac.jp )

Kokushikan University https://orcid.org/0000-0002-4973-3294

\section{Koichiro Yori}

Terumo Corporation

Keiichi Sugiura

Terumo Corporation

Makoto Jinno

Kokushikan University

\section{Research Article}

Keywords: Regenerative medicine, Cell processing, Media changing process, Injecting task, Machine learning

Posted Date: July 20th, 2021

DOI: https://doi.org/10.21203/rs.3.rs-713981/v1

License: (c) (i) This work is licensed under a Creative Commons Attribution 4.0 International License. Read Full License 


\section{Abstract}

In regenerative medicine, manual cell processing is labor-intensive and costly and needs to become more efficient. Recently, automatic cell culture systems equipped with vertically articulated robots have been developed. However, automating all cell processing tasks complicates these systems. This study aims to develop a simple and rational cell processing system by combining robot and human tasks. In a previous study, we improved the efficiency of discarding and injecting tasks using a robot in a media changing process. In this study, we further investigated the injection method and developed and evaluated two types of algorithms that are both accurate and can handle variable target volumes. One algorithm predicts the time to stop injecting based on the flow rate and corrects the stop timing based on the yintercept of the injection volume and the target volume. The other algorithm uses machine learning to predict the time to stop injecting based on the flow rate, $y$-intercept, and target volume. Our experimental results revealed the feasibility of both algorithms. With these algorithms, a robot can perform the injecting task more efficiently than a human.

\section{Introduction}

In recent years, cell transplantation has been used to repair damaged tissues. For example, skeletal myoblasts, mesenchymal stem cells, myocardial stem cells, embryonic stem cells, and induced pluripotent stem cells have been used to repair myocardial tissue damaged by ischemic heart diseases such as angina pectoris and myocardial infarction. Under these circumstances, a cell structure formed using a scaffold and a cell sheet, where cells form in a sheet shape, has been developed $[1,2]$.

Conventionally, these cells have been manufactured manually by an operator with specialized knowledge in a clean room called a cell processing center. The cost of manufacturing cells is high, and there is still room for improvement in its efficiency. In manual operation, there are individual differences in terms of operation time and contamination risk. Therefore, automatic cell culture apparatuses that use a robot arm to perform tasks related to cell culture have been developed [3-10]. Moreover, techniques related to cell culture automation have also been proposed [11-14]. Although automated injection has been promoted in fields other than regenerative medicine, accurately controlling the injection volume is difficult because teaching playback control is adopted $[15,16]$.

Our investigation revealed that most of the existing automatic cell culture apparatuses use single-arm or dual-arm robots, which can be used unattended [17]. However, the automation of all cell processing tasks complicates the system; the developed apparatuses lack flexibility (e.g., versatility and expandability), and the cost of the apparatuses is high. Because automatic cell culture apparatuses have been mainly developed by companies, such as machine manufacturers, they tend to be large-scale systems that automate all tasks or systems that use advanced technologies, such as robot arms.

The regenerative medicine products approved for manufacture and sale at this time have been approved by culture methods. Thus, a cell culture-assisting apparatus that collaborates with humans in a safety 
cabinet is considered easier to introduce than a fully automated apparatus. This study aims to develop a simple and rational cell processing system through the combination of the tasks performed by a robot

and those performed by a human. In a previous study, we proposed improving the efficiency of discarding and injecting tasks using a robot in a media changing process $[18,19]$. Such tasks have a high risk of liquid dripping and rely on the operator's experience.

In this study, we report the results of further investigation of the injection method and the development and evaluation of two types of algorithms that are both accurate and can handle variable target volumes. One algorithm predicts the time to stop injecting based on the flow rate and corrects the stop timing from the $y$-intercept of the injection volume and the target volume. The other algorithm uses machine learning $(\mathrm{ML})$ to predict the time to stop injecting based on the flow rate, y-intercept, and target volume. ML has been used for prediction and factor analysis in optical communications [20], and infectious disease epidemiology [21], and its effectiveness has been confirmed. However, there is no study on the application of $M L$ to the task of injecting a liquid. The application of $M L$ to the injecting task will reduce the necessity for manual data analysis, as in the previous study. Furthermore, if the learning model is simple, the amount of data required for learning should be minimal, allowing for highly accurate predictions. We conducted the preliminary experiments to calculate the parameters of the prediction equation for each algorithm. Afterward, we conducted evaluation experiments using the prediction equation to confirm the effectiveness of each algorithm. Our experimental results reveal that both algorithms are feasible and which algorithm is more suitable depending on the situation.

\section{Methods}

\section{Injection algorithm using $\Delta T$ and $X$}

The method for improving the efficiency of the injecting task is shown in the previous study [19]. Figure 1 shows the injecting task performed by a robot in this study. The angle of the injection bottle changed from $45^{\circ}$ to $70^{\circ}$ to reduce the culture media left in the injection bottle. The robot performs the injecting task only by rotating around the tool center point (TCP), the injecting spout of the injection bottle. To reduce the risk of liquid dripping, a protruding inspiration port on the lid of the injection bottle is changed to a non-protruding inspiration hole.

Figure 2 shows the transition of the injection volume after the start of the injection end motion. The injection algorithm predicts the injection volume $(X \%)$ to be injected in a fixed period $(\Delta T)$. If the injection is continued without stopping, the injection volume during $\Delta T$ is assumed to be $100 \%$. The injection end motion is triggered when the injection algorithm predicts that $X$ will reach the target volume. $\Delta T$ and $X$ depend on the injecting angle and the angular velocity of the injecting task, the structure of the lid of the injection bottle, and the control cycle of the robot. Therefore, it is necessary to determine $\Delta T$ and $X$ in advance by analyzing data obtained when the robot performs the injecting task.

In the previous study [19], a slope from 40 to $60 \mathrm{~g}$ was adopted, considering that the injection volume was fixed at $75 \mathrm{~g}$ and that the volume closer to the injection end motion could be more accurately predicted. 
However, the injection volume should be changed. The range slope, excluding $20 \mathrm{~g}$ at the start and end of the injection volume, was to be used since the flow rate is stable within the range, excluding the start and end of the injection.

\section{Correction by the y-intercept}

Although the conventional algorithm can achieve the same accuracy as the operator, it was found that the injection volume varied depending on the number of injections. The injection volume when the injection is repeated multiple times is shown in Fig. 3. Specifically, the remaining volume in the injection bottle decreases as the number of injections, and the following phenomenon occurs.

1. The y-intercept decreases (the $x$-intercept increases) because of the decrease in the volume injected during the injection start motion.

2. The final volume decreases because of the decrease in the volume injected during the injection end motion.

Therefore, the difference in (a) is used to compensate for the effect of (b). Figure 4 shows the system overview. First, $\Delta T$ and $X$ are calculated using load cell data when the injection experiment is repeated. The maximum volume of the culture media in the injection bottle is $500 \mathrm{~g}$. Because the injection bottle is tilted $70^{\circ}$ for injection, it is assumed that the remaining approximately $50 \mathrm{~g}$ cannot be injected. If the injection end motion is started when the injection volume exceeds $68 \mathrm{~g}$, approximately $75 \mathrm{~g}$ is injected. Thus, it is possible to inject six times in a row. The six times of this experiment was repeated six sets. The average value was adopted; $\Delta T$ was determined to be $1.28 \mathrm{~s}$, and $X$ was determined to be $75.0 \%$.

Afterward, the injection algorithm using the obtained $\Delta T$ and $X$ was applied to the original data. Because the data were obtained when the injection end motion was started after the injection volume exceeded 68 $\mathrm{g}$, it was possible to calculate a theoretical value for how much of a target volume should be set. An additional experiment with a target volume of $75 \mathrm{~g}$ can be conducted using the obtained $\Delta T$ and $\mathrm{X}$ to determine the difference between the theoretical and measured values; however, this was not conducted in this study. Figure 5 shows the correlation between the y-intercept of the injection volume and the difference between the theoretical and measured values. The smaller the y-intercept, the smaller the measured value, whereas the theoretical value remains constant. A linear approximation equation can be obtained because there is a linear correlation between the $y$-intercept and injection error. By calculating the volume of increase or decrease from the $y$-intercept and shifting the stop timing, it is possible to approach the theoretical value. The equation for calculating the correction volume $C_{1}$ using the yintercept $b_{y}$ is as follows.

$$
C_{1}=-0.0847\left(b_{y}-15.5478\right)
$$

A correction using the $x$-intercept is also possible. Figure 6 shows the correlation between the $x$-intercept of the injection volume and the difference between the theoretical and measured values. Each correction 
was applied to the original data to calculate the root mean square (RMS) of the difference between the theoretical and measured values. The result was 0.261 when the $y$-intercept was used and 0.262 when the $x$-intercept was used. Although both can be used, this study uses the y-intercept. By employing the correction by the $y$-intercept, it is possible to respond to changes in the injection volume caused by the number of injections.

\section{Correction by the target volume}

In Fig. 3, if the target volume is different, even if the y-intercept is the same, the effect of (b) is different because the remaining volume at the start of the injection end motion is different. The correction by the $y$ intercept is for a target volume of $75 \mathrm{~g}$. It includes the correction for the effect of (b). However, if the target volume is not $75 \mathrm{~g}$, the remaining volume at the start of the injection end motion increases or decreases compared to the case where the target volume is $75 \mathrm{~g}$. In other words, the effect of (b) changes and the correction of only (a) is insufficient. Therefore, the correction method for the case where the target volume is not $75 \mathrm{~g}$ is considered.

Figure 7 shows the correlation between the remaining volume at the start of the injection end motion and the injection volume after the injection end motion. The smaller the remaining volume, the smaller the injection volume. A linear approximation equation can be obtained because there is a linear correlation between the remaining volume and injection volume. Then, the change in injection volume per unit remaining volume can be calculated. Because the difference between $75 \mathrm{~g}$ and the target volume is the difference in the remaining volume, the equation for calculating the correction volume $C_{2}$ using the target volume $V_{t}$ is as follows.

$$
C_{2}=-0.0017\left(V_{t}-75.0\right)
$$

Using the correction by the target volume, the injection algorithm using $\Delta T$ and $X$ can be applied to target volumes other than $75 \mathrm{~g}$.

\section{Flowchart of the injection algorithm}

A flowchart of the injection algorithm using $\Delta T$ and $X$ with two types of corrections is shown in Fig. 8. In the conventional method, the injection end motion is started when the injection volume exceeds the stopping volume $V_{e}$. However, it turned out that an injection error occurred depending on the number of injections and target volume. Therefore, the correction volumes $C_{1}$ and $C_{2}$ are added to $V_{e}$. Although not employed in this study, another method for adding correction volumes $C_{1}$ and $C_{2}$ to the target volume $V_{t}$ is shown in Figure 9.

\section{Injection algorithm using machine learning}

As an alternative to the injection algorithm using $\Delta T$ and $\mathrm{X}$ with two types of corrections (called Algorithm A), the injection algorithm using $M L$ (called Algorithm B) is considered. 
Algorithm B uses multiple linear regression to predict the time to stop injecting based on the flow rate, the $y$-intercept of the injection volume, and the target value. When two features are fixed, there is a linear relationship between the remaining and predicted values. Therefore, linear regression is considered to be effective. Because the features include the $y$-intercept and target value, the correction of Algorithm $A$ is included. The prediction equation by the linear regression model is shown in Eq. (3), and each feature value of the linear regression model is shown in Fig. 10. The slope $x_{1}, y$-intercept $x_{2}$, and target value $x_{3}$ are feature values; the weights $w_{1}, w_{2}, w_{3}$, and the offset $b$ are parameters of the learned model; and the stopping volume $V_{e a}$ is a predicted value from the model. The model is developed using scikit-learn [22], a Python ML library.

$$
V_{e a}=w_{1} x_{1}+w_{2} x_{2}+w_{3} x_{3}+b
$$

First, preliminary experiments were repeated to collect training data, and a linear regression model was developed from the collected training data. For each target volume, six sets of injection experiments were conducted. The target volume was set between 50 and $150 \mathrm{~g}$ at $10 \mathrm{~g}$ intervals. The injection end motion began at $7 \mathrm{~g}$ less than the target volume to keep the final injection volume close to the target volume. 500 $\mathrm{g}$ of culture media was repeatedly injected. However, the remaining volume should not be less than $50 \mathrm{~g}$. Each parameter of Algorithm B was calculated using the training data. Algorithm A can also be expressed in Eq. (3). The values of each parameter for both algorithms are shown in Table 1. $V_{e a}$ in Fig. 8 can also be calculated using Algorithm B.

\section{Results And Discussion}

\section{Evaluation experiment}

Evaluation experiments were conducted to verify the effectiveness of each algorithm. For each target volume, two sets of evaluation experiments were conducted. The target volume was set between 50 and $150 \mathrm{~g}$ at $10-\mathrm{g}$ intervals. $500 \mathrm{~g}$ of culture media was repeatedly injected. However, the remaining volume should not be less than $50 \mathrm{~g}$. Table 1 shows the parameters used in the experiment.

Table 2, Table 3, and Fig. 11 show the evaluation experiment results. According to our previous study [19], the standard deviation $(3 \sigma)$ when the operator performed the injecting task was $1.5 \mathrm{~g}$, and the standard deviation $(3 \sigma)$ when the robot performed the injecting task using the conventional injection algorithm without correction was $1.18 \mathrm{~g}$. On the other hand, both Algorithms A and B showed a better standard deviation $(3 \sigma)$ than the conventional injection algorithm without correction for all target volumes. Therefore, both algorithms can be used, and which one depends on the situation. For example, if data for a certain target volume can only be collected and data analysis can be performed manually, Algorithm A is suitable. In contrast, if data for various target volumes can be collected and the data analysis cannot be performed manually, Algorithm B is suitable.

\section{Discussion on data used to calculate the parameters of Algorithm A}


Parameters calculated using the experimental data when the target volume was $75 \mathrm{~g}$ were used in the evaluation experiment of Algorithm A. The case to be considered is when the parameters are calculated using data from another target volume. Simulations are performed using preliminary ML experimental data to verify injection accuracy.

For comparison, the parameters were calculated using the experimental data when the target volume was $50 \mathrm{~g}$ or $150 \mathrm{~g}$. Calculating a theoretical value for how much of a target volume should be set was possible with these parameters. The difference between the theoretical and measured values was shown in Fig. 12. Although the results slightly differed depending on the data used to calculate the parameters, the difference was within $\pm 1.5 \mathrm{~g}$ for all target volumes.

\section{Discussion on data used to construct the model for Algorithm B}

Parameters calculated using all data with target volumes from $50 \mathrm{~g}$ to $150 \mathrm{~g}$ were used in the evaluation experiment of Algorithm B. The case to be considered is when the parameters are calculated using different training data. Simulations are performed using the preliminary ML experimental data to confirm injection accuracy.

For comparison, the parameters were calculated using the training data of four patterns. Each pattern has a different range and amount of training data. Calculating a theoretical value for how much of a target volume should be set was possible with these parameters. The difference between the theoretical and measured values was shown in Fig. 13. As shown in Fig. 13(a), Algorithm B achieved poor performance when experimental data with only a target volume of $75 \mathrm{~g}$ were used. For all other patterns, the difference was within $\pm 1.0 \mathrm{~g}$ for all target volumes.

\section{Conclusions}

Most of the existing automatic cell culture apparatuses are fully automated and lack flexibility. We aimed to develop a cell culture-assisting apparatus that collaborates with humans. In a previous study, we proposed an injection algorithm using a robot to improve the efficiency of the injecting task in the media changing process. In this study, we further investigated the injection method and developed two injection algorithms that are both accurate and can be applied to various target volumes. Evaluation experiments were conducted, and the following results were obtained.

1. The injection accuracy was improved by adding a correction using the y-intercept of the injection volume and the target volume to the conventional injection algorithm.

2. A linear regression model was developed by $M L$ using the flow rate, y-intercept, and target value as training data. The injection accuracy using the linear regression model was equivalent to that of the injection algorithm with the correction.

3. Both injection algorithms were applicable to target volumes ranging from 50 to $150 \mathrm{~g}$. 
Thus, it was determined that the robot could perform the injecting task more efficiently than the operator. Which algorithm to use depends on the purpose. The choice can be made based on the amount and type of data collected and the effort required analyzing the data. In the future, we will incorporate the new injection algorithm into the injecting robot and optimize the task procedures in combination with the discarding robot.

\section{Abbreviations}

ML: Machine learning

TCP: Tool center point

RMS: Root mean square

\section{Declarations}

\section{Availability of data and materials}

Not applicable.

\section{Competing interests}

Research supported by Terumo Corporation.

\section{Funding}

Not applicable.

\section{Authors' contributions}

$\mathrm{RN}$ conducted the research and drafted the manuscript. KY and KS provided requirement specifications and their knowledge on cell processing and designed the lid of the injection bottle. MJ supervised the research and reviewed the manuscript. All authors read and approved the final manuscript.

\section{Acknowledgements}

Not applicable.

\section{References}

1. Imamura T, Kinugawa K, Sakata Y, Miyagawa S, Sawa Y, Yamazaki K, Ono M (2016) Improved clinical course of autologous skeletal myoblast sheet (TCD-51073) transplantation when compared to a propensity score-matched cardiac resynchronization therapy population. J Artif Organs 19(1):8086. 
2. Sawa Y, Yoshikawa Y, Toda K, Fukushima S, Yamazaki K, Ono M, Sakata Y, Hagiwara N, Kinugawa K, Miyagawa S (2015) Safety and efficacy of autologous skeletal myoblast sheets (TCD-51073) for the treatment of severe chronic heart failure due to ischemic heart disease. Circ J 79(5):991-999.

3. Kempner M, Felder R (2002) A review of cell culture automation. JALA 7(2):56-62.

4. Bernard C, Connors D, Barber L, Jayachandra S, Bullen A, Cacace A (2004) Adjunct automation to the cellmate cell culture robot. JALA 9(4):209-217.

5. Thomas RJ, Chandra A, Hourd PC, Williams DJ (2008) Cell culture automation and quality engineering. JALA 13(3):152-158.

6. Liu Y, Hourd P, Chandra A, Williams DJ (2010) Human cell culture process capability: a comparison of manual and automated production. J Tissue Eng Regen M 4:45-54.

7. Jain S, Sondervan D, Rizzu P, Bochdanovits Z, Caminada D, Heutink P (2011) The complete automation of cell culture: improvements for high-throughput and high-content screening. J Biomol Screen 16(8):932-939.

8. Schultz A, Koch S, Fuss M, Mazzotta AS, Sarzotti-Kelsoe M, Ozaki DA, Montefiori DC, von Briesen H, Zimmermann H, Meyerhans A (2012) An automated HIV-1 Env-pseudotyped virus production for global HIV vaccine trials. PLoS ONE 7(12). doi:10.1371/journal.pone.0051715

9. Nakashima K, Kanazawa H, Sakurai T, Hasunuma H, Sato O (2014) Automated cell culture system for stem cells. Seibutsu-kogaku Kaishi, 92(9):473-478 (in Japanese).

10. Ochs J, Biermann F, Piotrowski T, Erkens F, Nießing B, Herbst L, König N, Schmitt RH (2021) Fully Automated Cultivation of Adipose-Derived Stem Cells in the StemCellDiscovery-A Robotic Laboratory for Small-Scale, High-Throughput Cell Production Including Deep Learning-Based Confluence Estimation. Processes 9(4):575. doi:10.3390/pr9040575.

11. Kino-oka M, Chowdhury SR, Muneyuki Y, Manabe M, Saito A, Sawa Y, Taya M (2009) Automating the expansion process of human skeletal muscle myoblasts with suppression of myotube formation. Tissue Eng Part C Methods 15(4):717-728.

12. Shu K, Kiyama M, Nozaki T, Nishimura A, Suzuki D, Kato M, Igarashi $Y$, and Takeda S (2016) Wider adoption of regenerative medicine driven by open innovation. Hitachi Review 64(10):693-698.

13. Lehmann R, Severitt JC, Roddelkopf T, Junginger S, Thurow K (2016) Biomek Cell Workstation: a variable system for automated cell cultivation. J Lab Autom 21:439-450.

14. Moutsatsou P, Ochs J, Schmitt RH, Hewitt CJ, Hanga MP (2019) Automation in cell and gene therapy manufacturing: From past to future. Biotechnol Lett 41:1245-1253. doi:10.1007/s10529-019-02732$z$.

15. Lindsay W (1983) Automatic pouring and metal distribution systems. Foundry Trade J:151-176.

16. Noda Y, Terashima K (2006) Flow rate model for tilting-type automatic pouring system and flow rate feedforward control by using inverse model. Transactions of the Japan Society of Mechanical Engineers Series C 72(722):3147-3154 (in Japanese). 
17. Shimuzu M, Yori K, Sameshima T, Nonoyama R, Jinno M (2018) Challenges faced by the existing automated culture device for regenerative medicine business. The Japanese Journal of Medical Instrumentation 88(4):433-439. doi:10.4286/jjmi.88.433 (in Japanese).

18. Nonoyama R, Jinno M, Sameshima T, Yori K (2018) Study on cell culture processing system to improve task efficiency (System concept and efficiency improvement of the discarding task using a robot in a media change process). Transactions of the JSME 84(859). doi:10.1299/transjsme.1700497 (in Japanese).

19. Nonoyama R, Yori K, Sameshima T, Jinno M (2019) Study on cell culture processing system to improve task efficiency (Efficiency improvement of the injecting task using a robot in a media change process). Transactions of the JSME 85(874). doi:10.1299/transjsme.18-00421 (in Japanese).

20. Nyarko-Boateng O, Adekoya AF, Weyori BA (2021) Predicting the actual location of faults in underground optical networks using linear regression. Engineering Reports 3(3). doi:10.1002/eng2.12304

21. Roy S, Ghosh P (2020) Factors affecting COVID-19 infected and death rates inform lockdown-related policymaking. PLoS ONE 15(10). doi:10.1371/journal.pone.0241165

22. Pedregosa F, Varoquaux G, Gramfort A, Michel V, Thirion B, Grisel O, Blondel M, Prettenhofer P, Weiss R, Dubourg V, Vanderplas J, Passos A, Cournapeau D, Brucher M, Perrot M, Duchesnay É (2011) Scikit-learn: Machine Learning in Python. J Mach Learn Res 12:2825-2830.

\section{Tables}

Table. 1 Parameters for the Evaluation Experiment of Algorithm A and Algorithm B.

\begin{tabular}{|lll|}
\hline Parameters & Algorithm A & Algorithm B \\
\hline$w_{1}$ & -0.9634 & -0.8120 \\
\hline$w_{2}$ & -0.0847 & -0.1421 \\
\hline$w_{3}$ & 0.9983 & 1.0007 \\
\hline$b$ & -1.1894 & -3.5116 \\
\hline
\end{tabular}

Table. 2 Experimental results of Algorithm A. 


\begin{tabular}{|lllll|}
\hline $\begin{array}{l}\text { Target } \\
\text { volume [g] }\end{array}$ & $\begin{array}{l}\text { RMS of injection } \\
\text { error [g] }\end{array}$ & $\begin{array}{l}\text { Average } \\
{[\mathbf{g}]}\end{array}$ & $\begin{array}{l}\text { Standard deviation } \\
(\mathbf{1 \sigma}) \mathbf{g}]\end{array}$ & $\begin{array}{l}\text { Standard deviation } \\
\mathbf{( 3 \sigma})[\mathbf{g}]\end{array}$ \\
\hline 50 & 0.44 & 50.24 & 0.38 & 1.13 \\
60 & 0.27 & 60.18 & 0.21 & 0.62 \\
\hline 70 & 0.40 & 70.17 & 0.38 & 1.13 \\
80 & 0.37 & 80.18 & 0.34 & 1.01 \\
90 & 0.32 & 90.24 & 0.23 & 0.69 \\
100 & 0.25 & 100.13 & 0.23 & 0.70 \\
110 & 0.24 & 110.08 & 0.24 & 0.72 \\
120 & 0.23 & 120.15 & 0.18 & 0.54 \\
130 & 0.20 & 129.88 & 0.18 & 0.55 \\
140 & 0.27 & 139.86 & 0.25 & 0.75 \\
150 & 0.31 & 149.91 & 0.32 & 0.97 \\
\hline
\end{tabular}

Table. 3 Experimental results of Algorithm B.

\begin{tabular}{|lllll|}
\hline $\begin{array}{l}\text { Target } \\
\text { volume [g] }\end{array}$ & $\begin{array}{l}\text { RMS of injection } \\
\text { error [g] }\end{array}$ & $\begin{array}{l}\text { Average } \\
\mathbf{[ g ]}\end{array}$ & $\begin{array}{l}\text { Standard deviation } \\
\mathbf{( 1 \sigma )}[\mathbf{g}]\end{array}$ & $\begin{array}{l}\text { Standard deviation } \\
\mathbf{( 3 \sigma}) \mathbf{g}]\end{array}$ \\
\hline 50 & 0.37 & 50.18 & 0.34 & 1.01 \\
\hline 60 & 0.24 & 59.97 & 0.25 & 0.74 \\
\hline 70 & 0.34 & 70.15 & 0.32 & 0.97 \\
\hline 80 & 0.30 & 79.95 & 0.32 & 0.95 \\
\hline 90 & 0.28 & 90.06 & 0.29 & 0.87 \\
\hline 100 & 0.32 & 99.89 & 0.32 & 0.96 \\
\hline 110 & 0.29 & 110.11 & 0.28 & 0.85 \\
\hline 120 & 0.21 & 120.10 & 0.20 & 0.60 \\
\hline 130 & 0.30 & 129.94 & 0.33 & 0.98 \\
\hline 140 & 0.35 & 140.05 & 0.37 & 1.12 \\
\hline 150 & 0.29 & 150.04 & 0.32 & 0.95 \\
\hline
\end{tabular}

Figures 


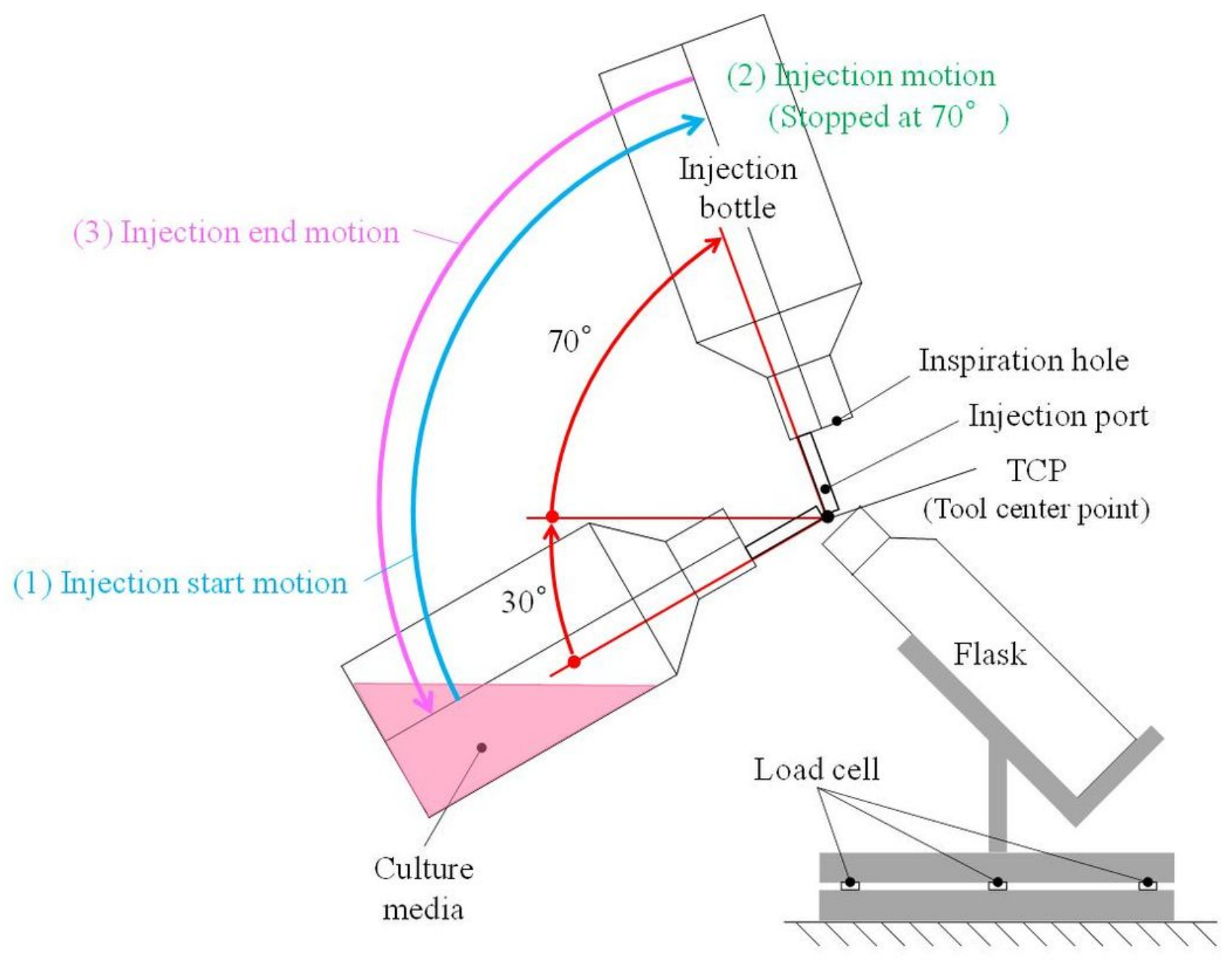

\section{Figure 1}

Injecting task performed by the robot. (1) The robot rotates the injection bottle and injects the culture media into a flask. (2) The robot stops at $70^{\circ}$ and continues to inject. (3) The robot returns to the initial position and finishes the injecting task. 


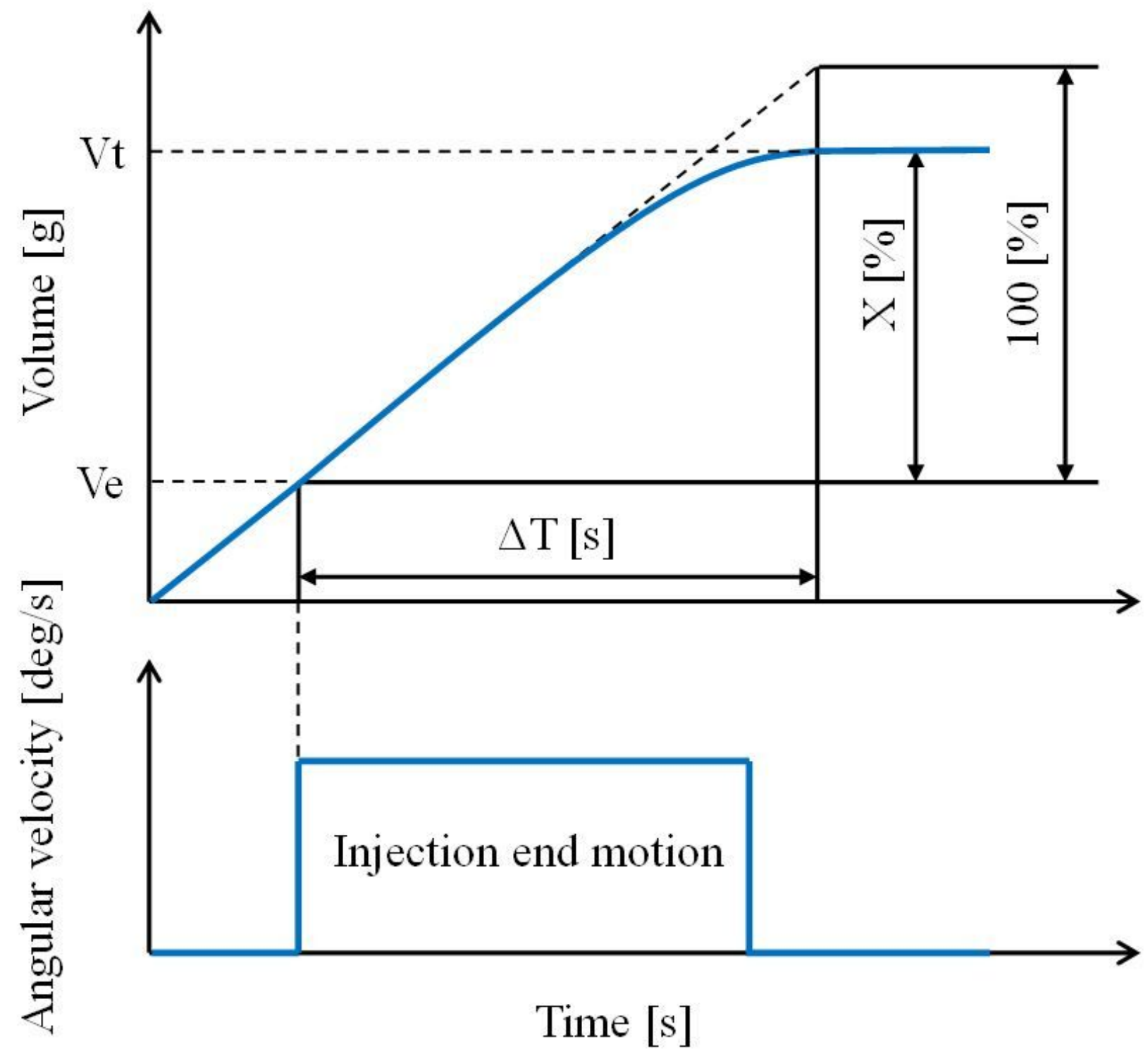

Figure 2

Transition of the injection volume after the start of the injection end motion. To inject the target volume $\mathrm{Vt}$, the injection end motion is started when the injection volume exceeds the stop volume Ve. 


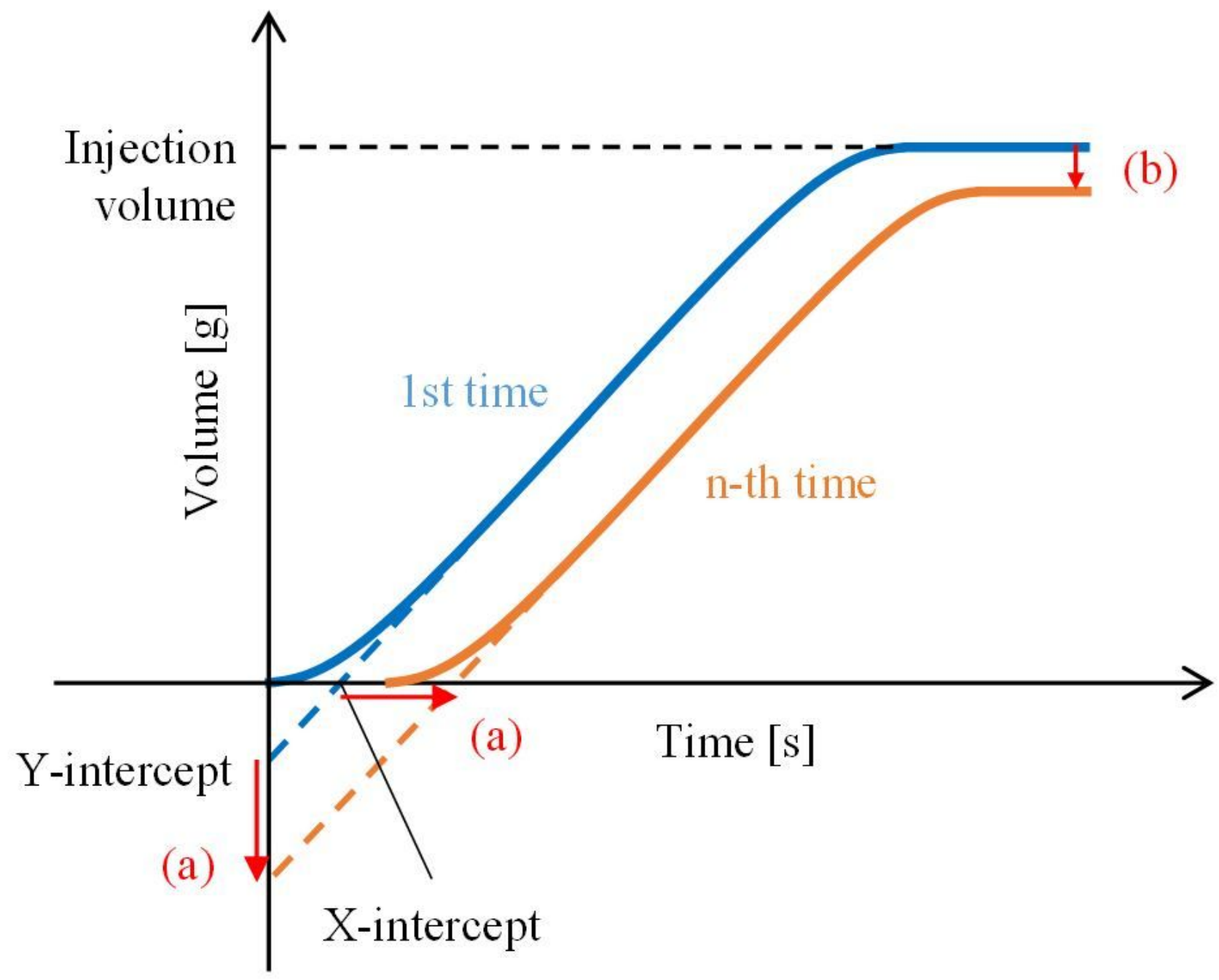

Figure 3

The injection volume when the injection is repeated multiple times. The injection volume varies depending on the number of injections. 


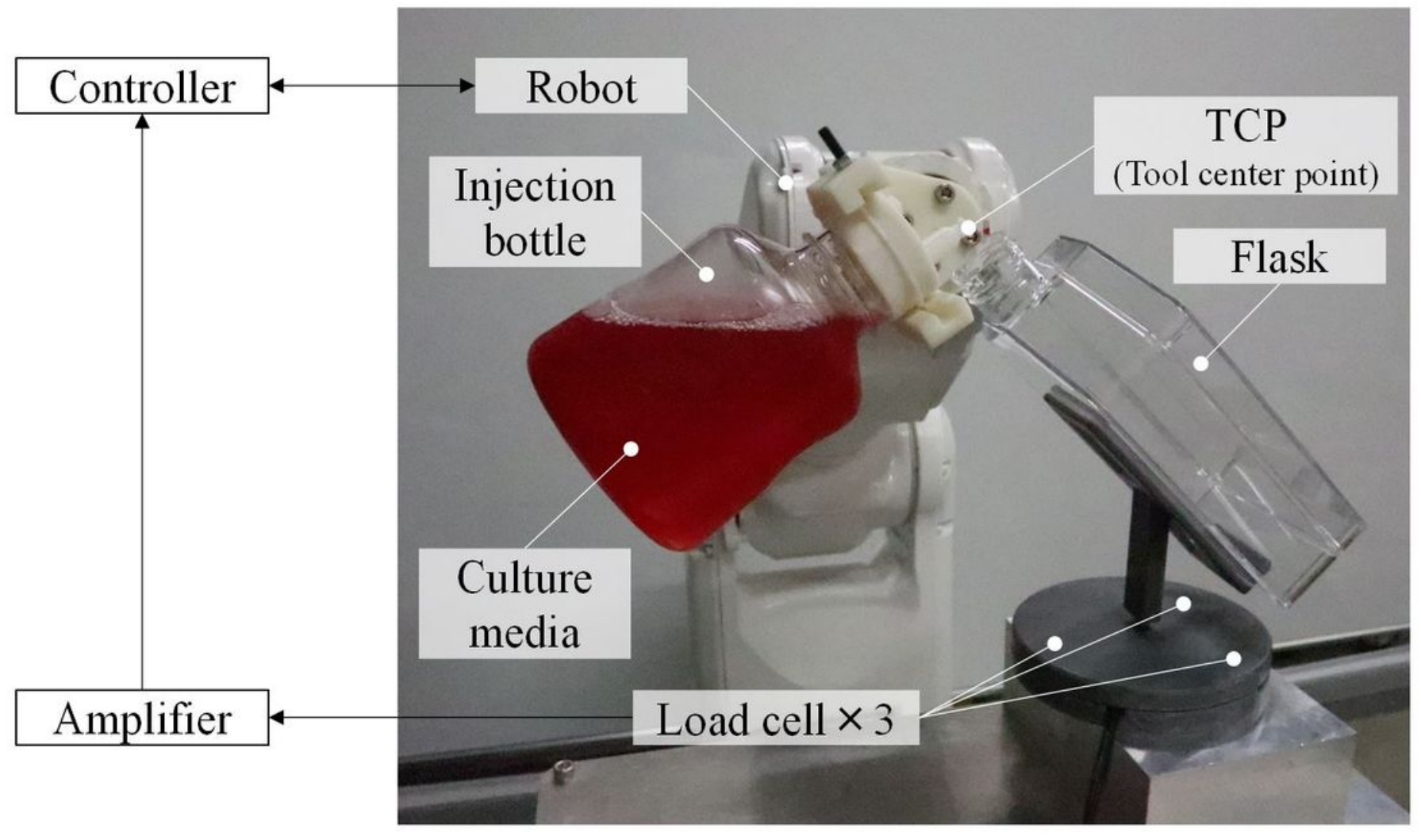

Figure 4

System overview. The injection was performed only by rotating the 6th axis of a 6-axis articulated robot. 


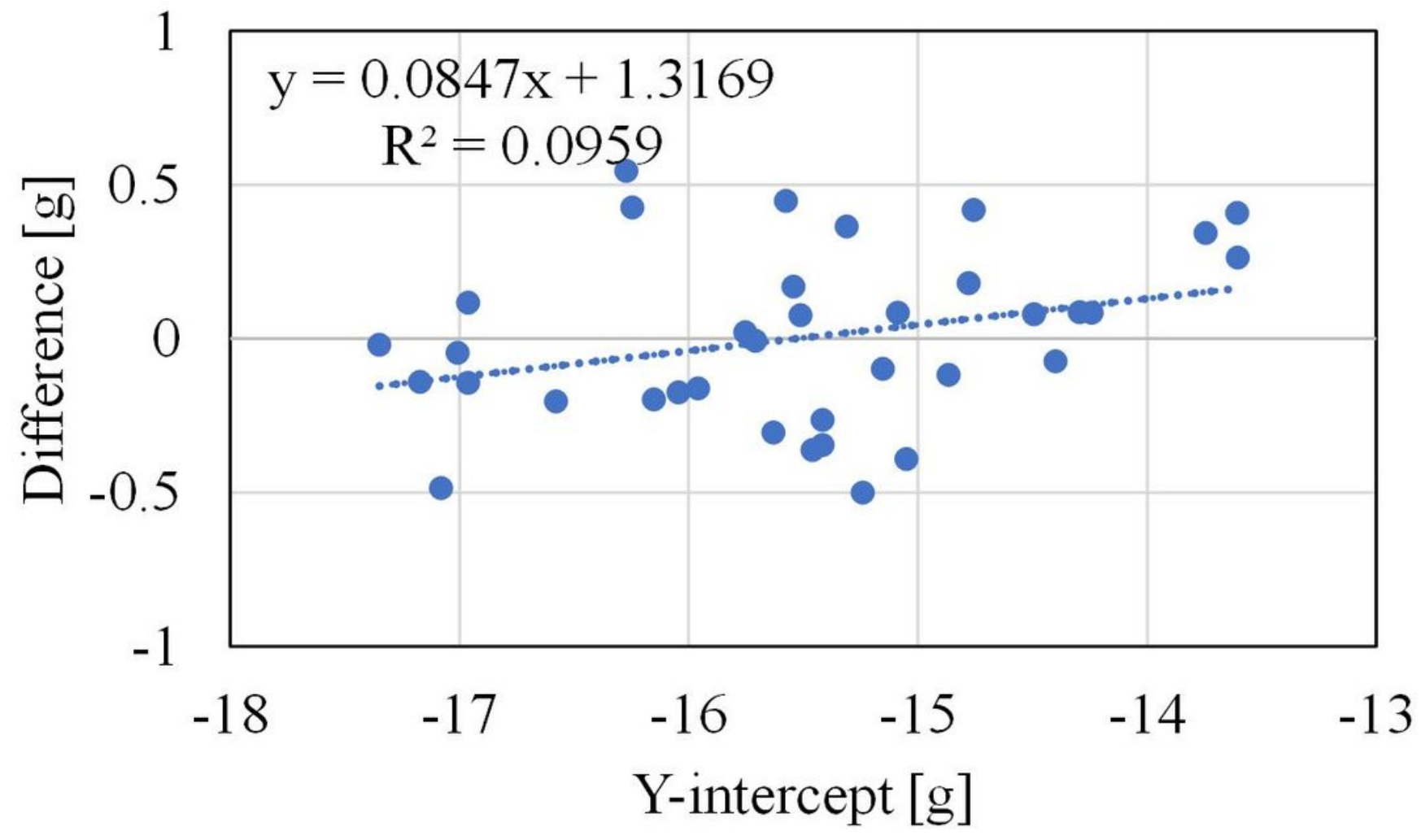

Figure 5

Correlation between the y-intercept of the injection volume and the difference between the theoretical and measured values. The measured value decreases as the $y$-intercept decreases, whereas the theoretical value remains constant. 


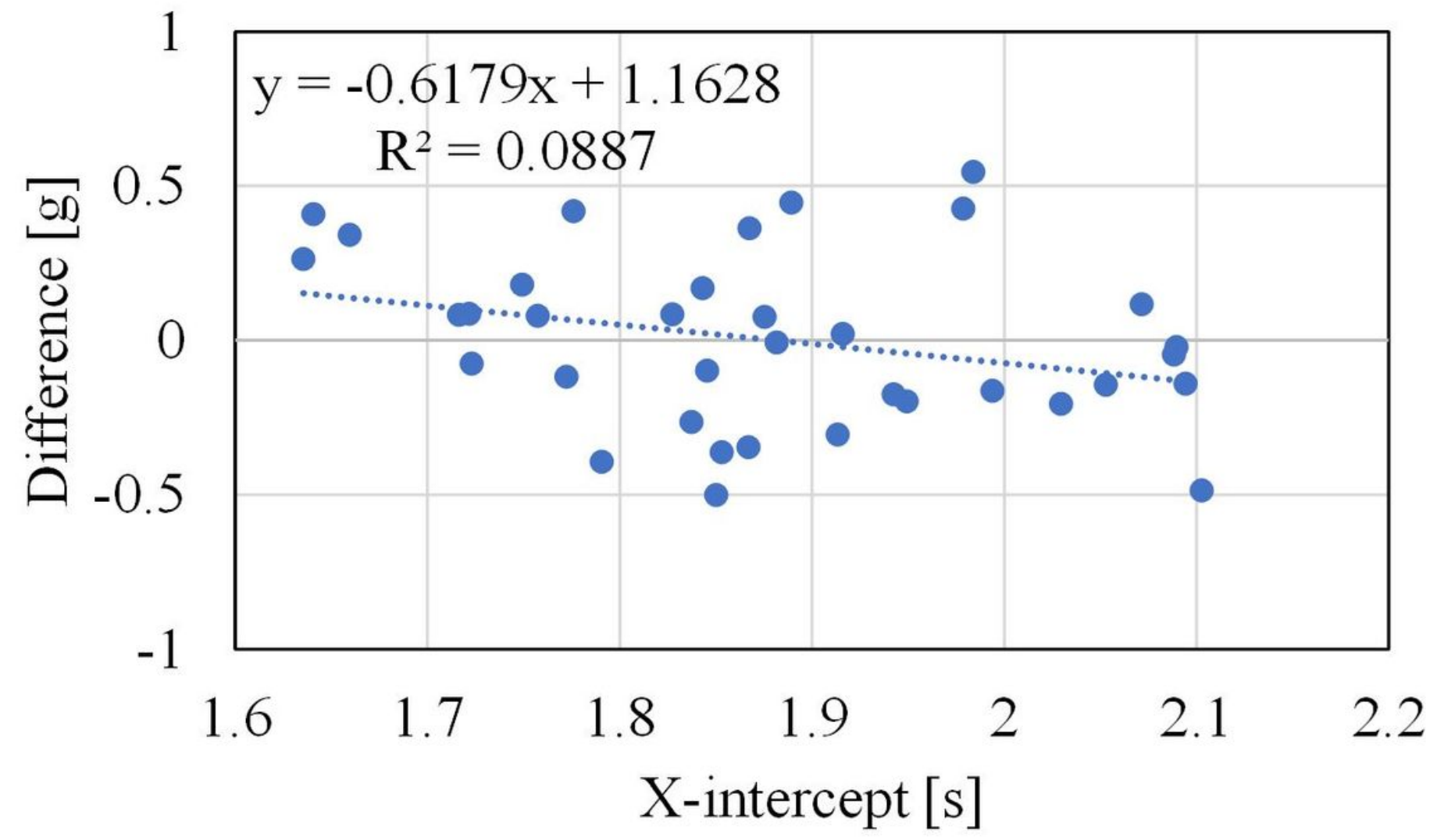

Figure 6

Correlation between the x-intercept of the injection volume and the difference between the theoretical and measured values. The measured value decreases as the $x$-intercept increases, whereas the theoretical value remains constant. 


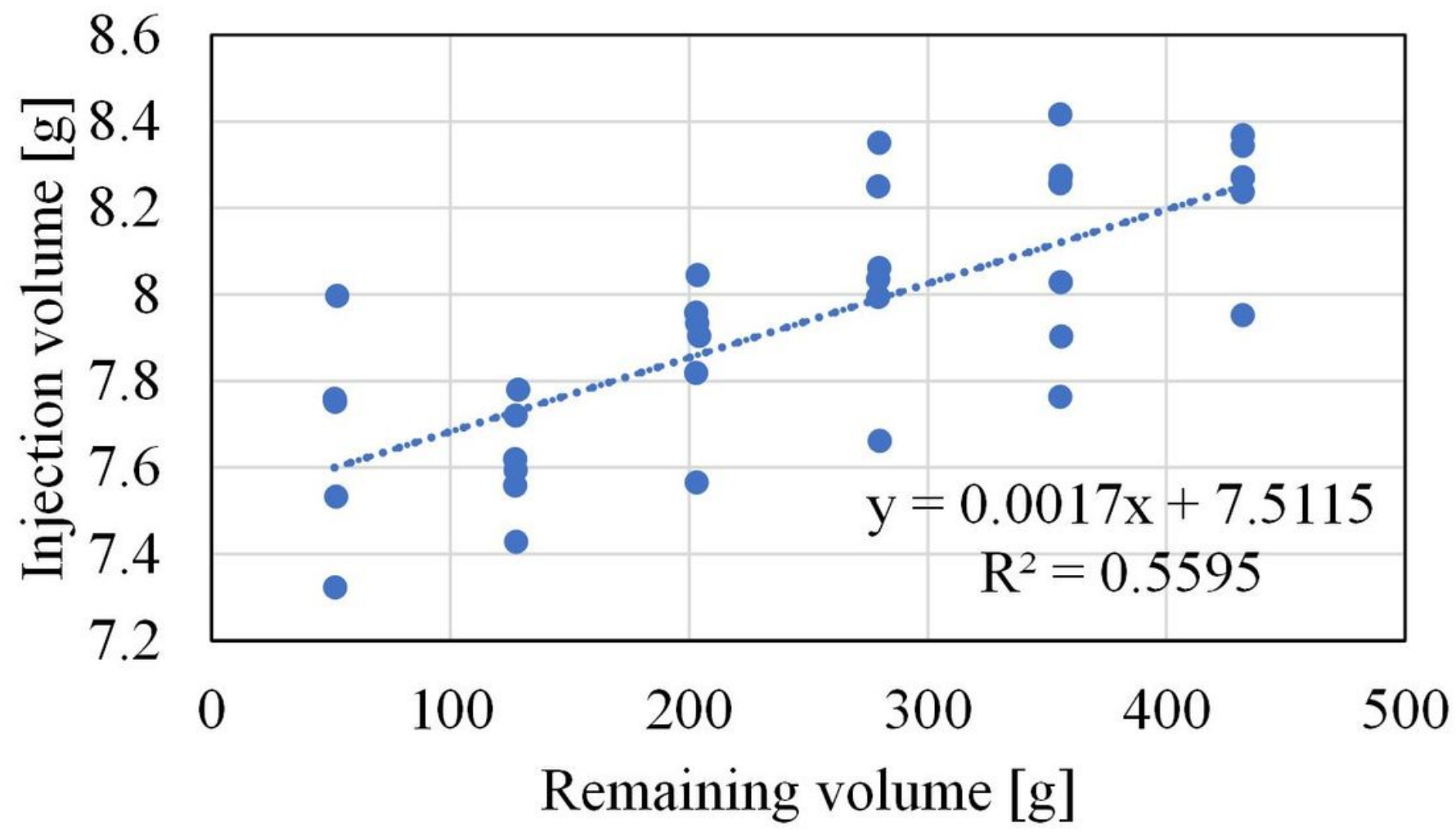

Figure 7

Correlation between the remaining volume in the injection bottle and the injection volume after the start of the injection end motion. The injection volume decreases as the remaining volume decreases. 


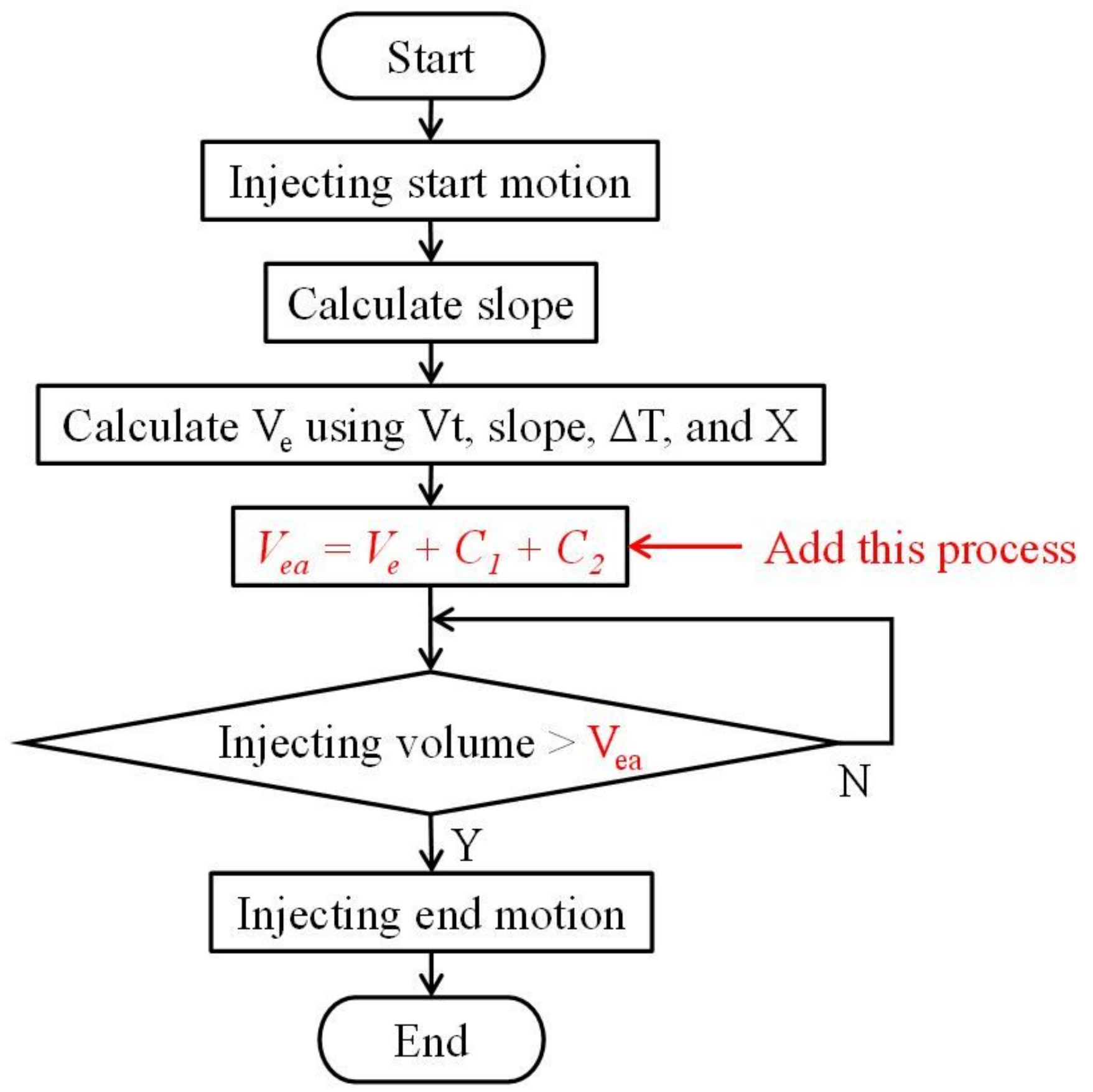

Figure 8

Flowchart of the injection algorithm using $\Delta \mathrm{T}$ and $\mathrm{X}$ with two types of correction. The correction volume $\mathrm{C} 1$ and $\mathrm{C} 2$ are added to $\mathrm{Ve}$. 


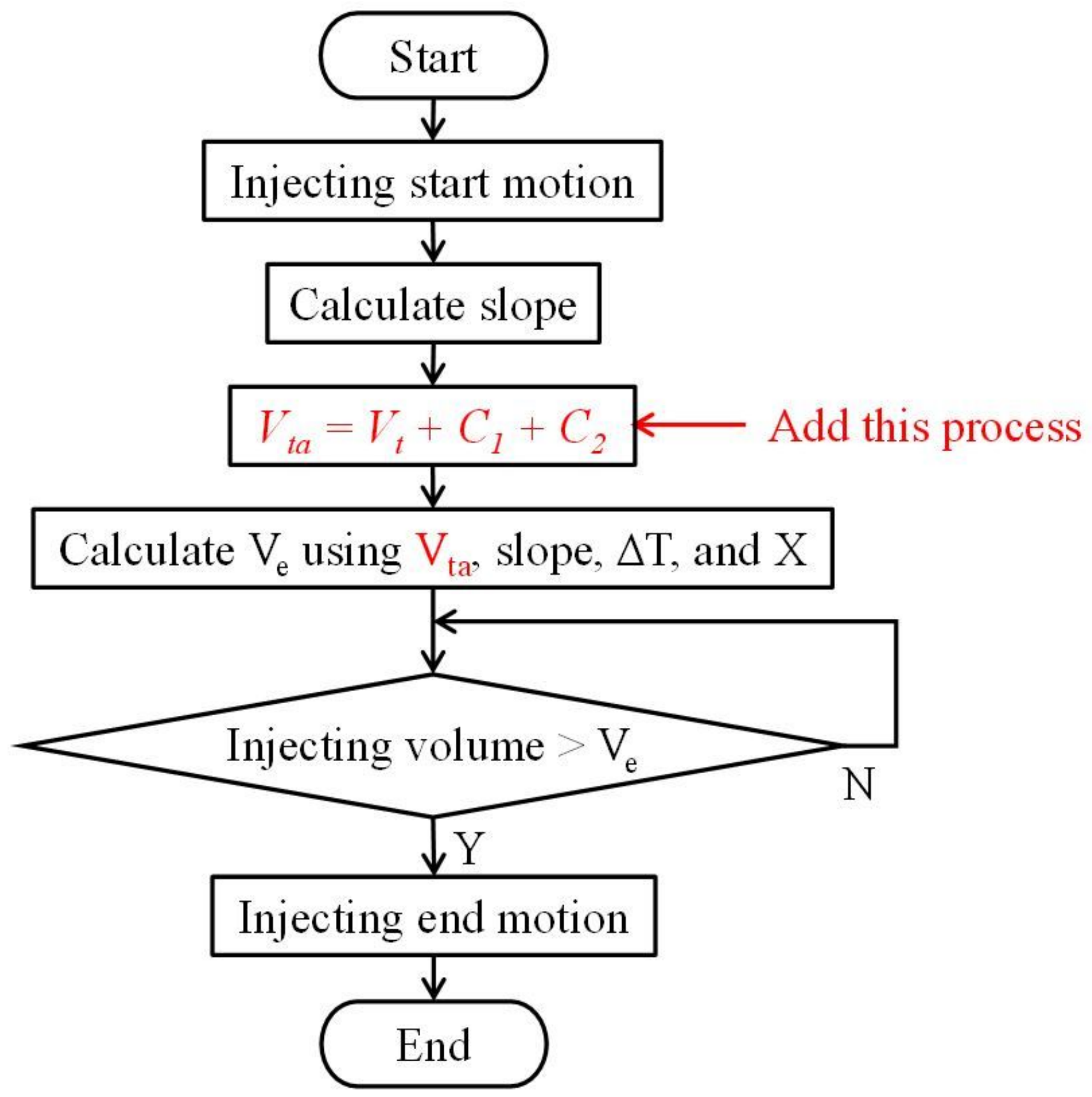

Figure 9

Flowchart of the injection algorithm using $\Delta \mathrm{T}$ and $\mathrm{X}$ with two types of correction. The correction volume $\mathrm{C} 1$ and $\mathrm{C} 2$ are added to $\mathrm{Vt}$. 


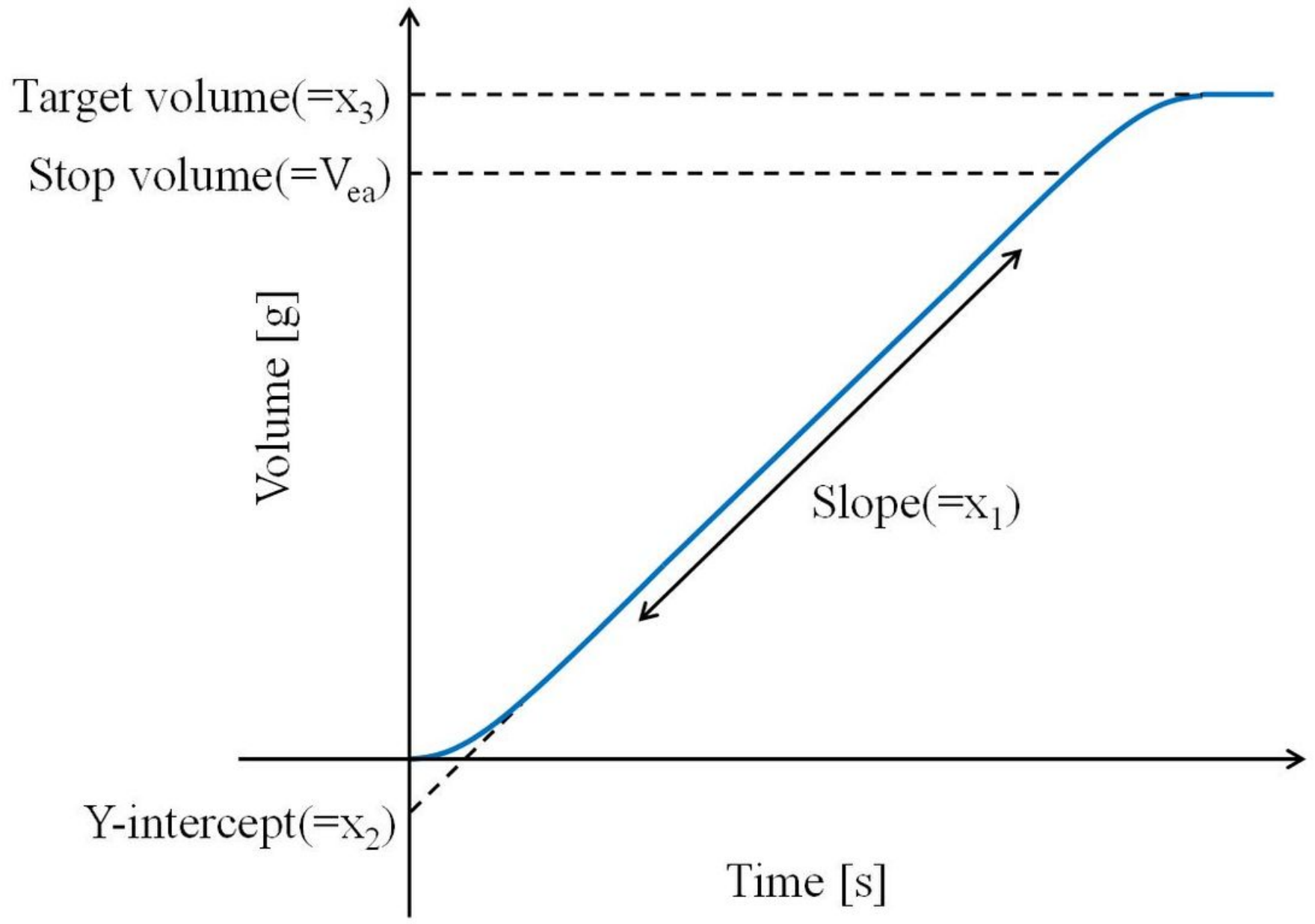

Figure 10

Each feature value of the linear regression model. The slope $x 1, y$-intercept $x 2$, and target volume $x 3$ can be used to calculate the stop volume Vea.

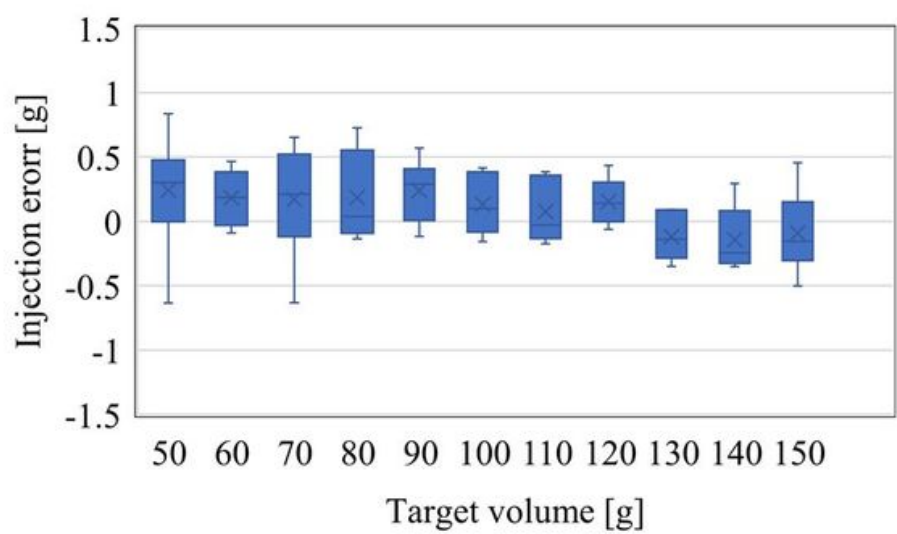

(a)

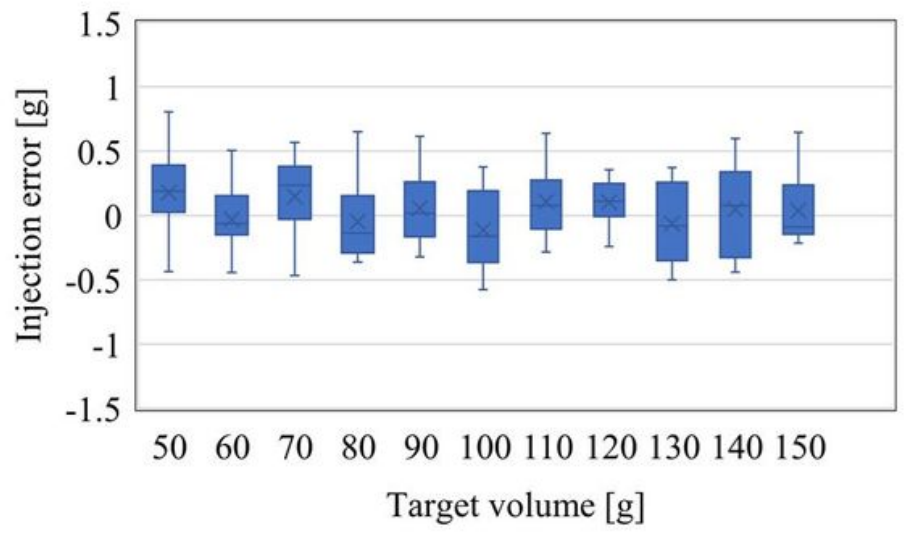

(b) 
Figure 11

The injection error for each target volume: (a) Algorithm A; (b) Algorithm B.

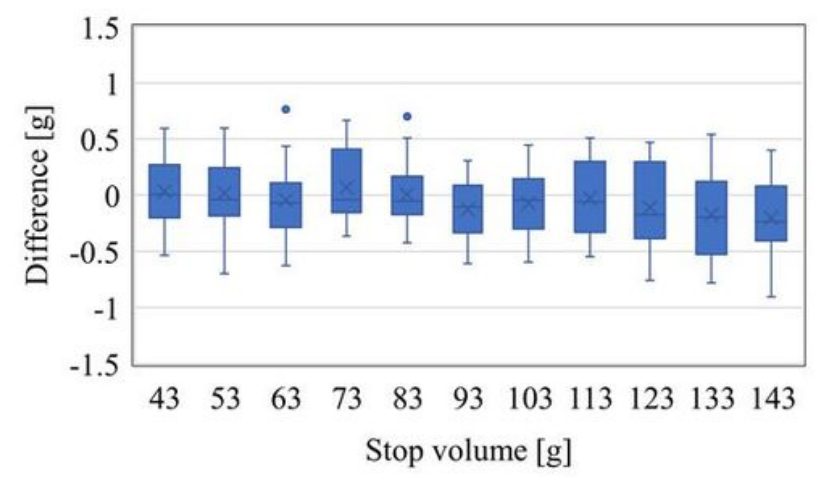

(a)

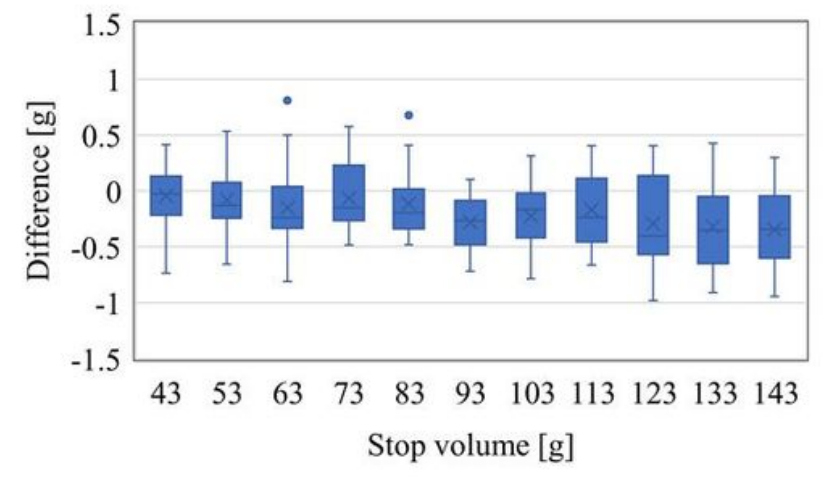

(b)

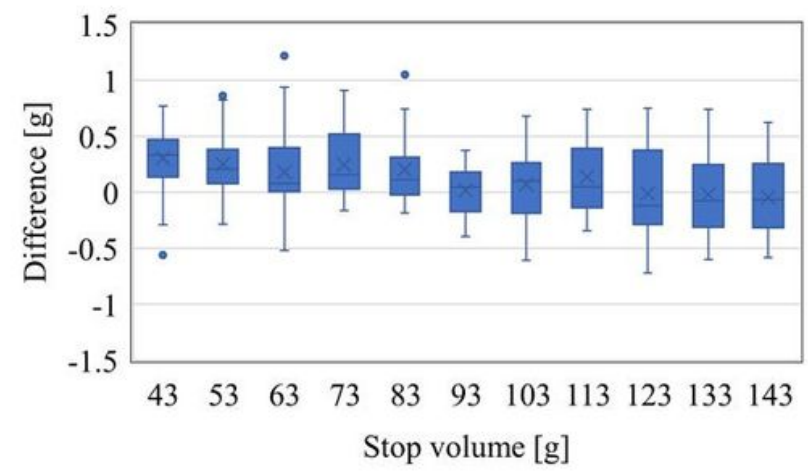

(c)

\section{Figure 12}

The difference between the theoretical and measured values for each stops volume. To calculate the parameters of Algorithm A, experimental data for various target values were used: (a) The target volume is $75 \mathrm{~g}$; (b) the target volume is $50 \mathrm{~g}$; (c) the target volume is $150 \mathrm{~g}$. 


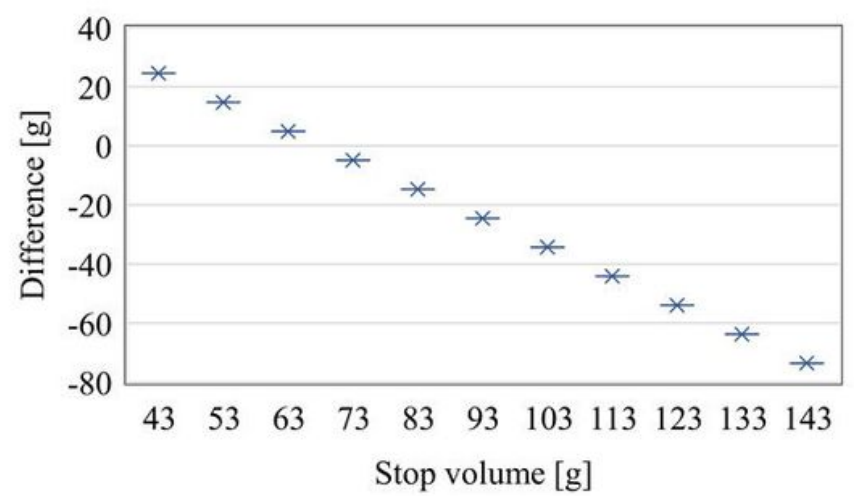

(a)

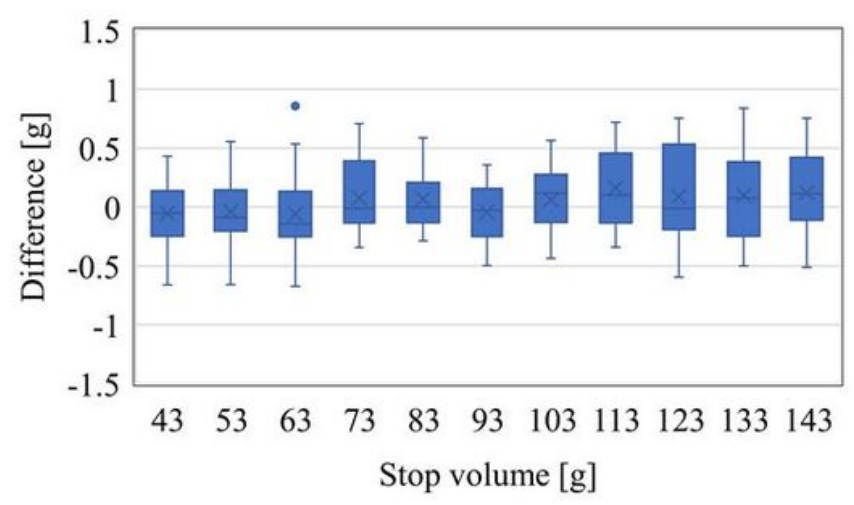

(c)

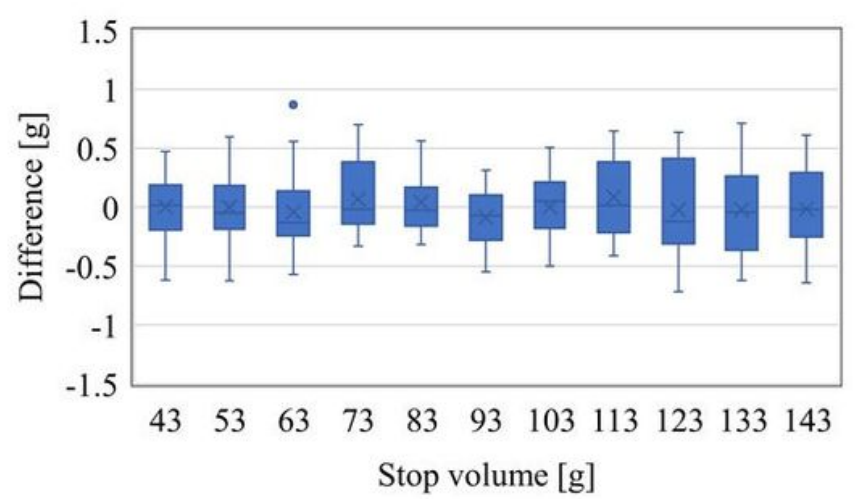

(b)

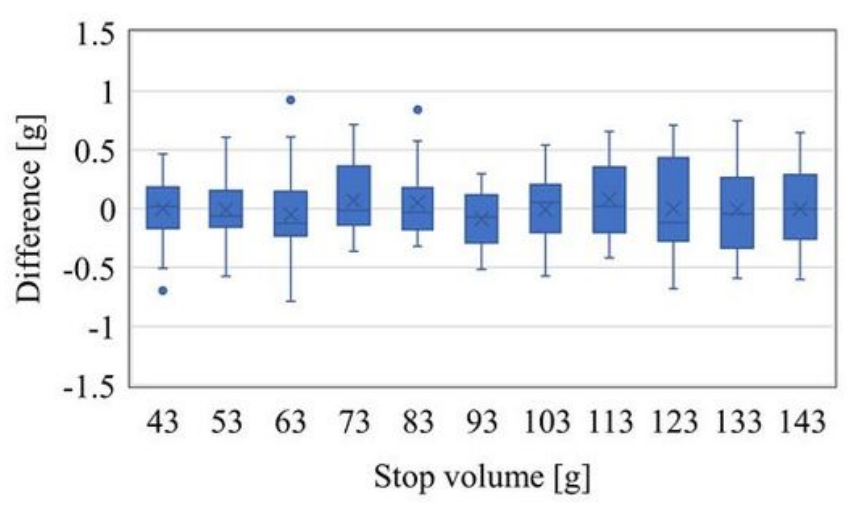

(d)

\section{Figure 13}

The difference between the theoretical and measured values for each stops volume. Machine learning was performed using a variety of training data: (a) Six sets of data with a target volume of $75 \mathrm{~g}$; (b) six sets of data with target volumes from 50 to $150 \mathrm{~g}$ at 10-g intervals; (c) one set of data with target volumes from 50 to $150 \mathrm{~g}$ at 10-g intervals; (d) six sets of data with target volumes of 50 and $150 \mathrm{~g}$. 